\title{
Flavon exchange effects in models with abelian flavor symmetry
}

\author{
Ilja Dorsner and S.M. Barr \\ Bartol Research Institute \\ University of Delaware \\ Newark, DE 19716
}

October 30, 2018

\begin{abstract}
In models with abelian flavor symmetry the small mixing angles and mass ratios of quarks and leptons are typically given by powers of small parameters characterizing the spontaneous breaking of flavor symmetry by "flavon" fields. If the scale of the breaking of flavor symmetry is near the weak scale, flavon exchange can lead to interesting flavor-violating and $\mathrm{CP}$ violating effects. These are studied. It is found that $d_{e}, \mu \rightarrow e+\gamma$, and $\mu$-e conversion on nuclei can be near present limits. For significant range of parameters $\mu-e$ conversion can be the most sensitive way to look for such effects.
\end{abstract}




\section{Introduction}

Flavor symmetry was first proposed to explain the structure of the quark and lepton mass spectrum and the CKM mixing of the quarks [1, 2]. More recently these ideas have been extended to account for the observed patterns of neutrino masses and mixings [3]. In the context of supersymmetry, flavor symmetry has been invoked to solve the problem of flavor changing neutral currents, i.e. "the SUSY Flavor Problem" [4, 5].

A wide assortment of flavor symmetries has been suggested. In particular, models based on both non-abelian and abelian symmetries have been constructed. One virtue of non-abelian symmetries is that they can lead to degenerate masses, which have various theoretical uses. For example, one solution to the SUSY flavor problem is to posit a near degeneracy of the squark/slepton masses of the first two families. For another example, large neutrino mixing angles can be obtained by positing nearly degenerate neutrino masses. However, in this paper we shall be interested in abelian flavor symmetries.

The main use to which abelian symmetries have been put is to explain the hierarchical patterns of quark and lepton masses and mixings. It has been observed that all of the interfamily mass ratios and mixing angles can be written as powers of one or two small parameters. For example, the quark mass ratios and mixings can be written $V_{u s} \sim \epsilon, V_{c b} \sim \epsilon^{2}, V_{u b} \sim \epsilon^{3}$, $m_{d} / m_{s} \sim \epsilon^{2}, m_{s} / m_{b} \sim 0.4 \epsilon^{2}$, and so on, where $\epsilon \sim \lambda \sim 0.2$, the Wolfenstein parameter [6]. This has suggested to many theorists the idea that there is a weakly broken abelian symmetry which distinguishes fermions that are of the same type but of different families. Suppose, for instance, that there is a $U(1)_{F}$ flavor symmetry, under which the Standard Model Higgs has charge zero, the fermions $\psi_{i}^{c}$ and $\psi_{j}$ have charges $\bar{q}_{i}$ and $q_{j}$, and a "flavon" field $S$ has charge -1 . Then a Yukawa operator $\psi_{i}^{c} \psi_{j} H$ is forbidden by the flavor symmetry, but the effective operator $\psi_{i}^{c} \psi_{j} H\left(S / M_{F}\right)^{\left(\overline{q_{i}}+q_{j}\right)}$ is not. Such an effective operator might arise from integrating out fields whose mass is of order $M_{F}$, the "flavor scale". If one assumes that the breaking of $U(1)_{F}$ is weak, in the sense that $\langle S\rangle / M_{F}=\epsilon \ll 1$, then one has explained the fact that the effective mass parameter of the term $\psi_{i}^{c} \psi_{j}$ is proportional to a power of the small quantity $\epsilon$. This is the basic idea of Froggatt and Nielson [1], which has inspired a very large number of models in the literature.

Aside from having the potential to explain the hierarchies observed among fermion masses and mixing angles, this idea can be used to construct models 
in which the dangerous flavor-changing effects in supersymmetric models are suppressed by "flavor alignment" [4]. The idea here is that in the preferred basis defined by the abelian flavor charge assignments the off-diagonal elements of both the fermion mass matrices and the sfermion mass-squared matrices are suppressed by powers of the small parameters which characterize flavor breaking (i.e. parameters like $\epsilon$ ). Thus the fermion and sfermion mass matrices are nearly "aligned" by flavor symmetry. The angles expressing their misalignment are suppressed by powers of the small parameters. If this suppression is strong enough it would solve the SUSY Flavor Problem.

In this paper we examine some of the possible consequences for phenomenology of the exchange of the "flavon" fields themselves. A point that should be stressed from the outset is that there do not have to be such consequences at all. The reason is that the flavor scale $M_{F}$ can be anything from the weak scale up to the Planck scale. All that matters is that the ratio $\langle S\rangle / M_{F}$ of the flavon expectation value (or values) to the flavor scale be somewhat smaller than 1. If the flavor scale is much larger than the weak scale, then the phenomenological effects of flavon exchange will be unobservably small. In fact, many papers assume that the flavor scale is near the Planck scale, which is certainly a reasonable expectation. However, since we do not know a priori what the flavor scale is, it is interesting to investigate the phenomenology that would result from its being near the weak scale, and in particular to ask how low the flavor scale could actually be given present limits on flavor-changing and CP-violating processes. We would also like to know in which processes flavon-exchange effects would be likely first to show up.

There are many ways that new flavor physics just above the weak scale could affect low-energy phenomenology. For instance, if the abelian flavor group is local, the exchange of the corresponding gauge bosons could cause flavor-changing neutral current processes. We will assume that the flavor group is either global, or breaks to a global symmetry at a sufficiently high scale that such gauge-boson-exchange effects can be ignored. We are only interested in this paper in the exchange of the flavon fields themselves.

There are many models with abelian flavor symmetry, and the number of parameters in such models can be large. What we shall do, therefore, is write down an effective low-energy theory that has a managably small number of parameters and that has some of the typical features of models with abelian flavor symmetry. Studying this toy model will give some idea of the likely magnitude of various effects. We will then look at some variations of the 
model to see how they would change the conclusions.

\section{A simple effective theory of flavon physics}

The model we shall study has a single flavon field $S$ that is a singlet under the Standard Model gauge group. The effective Yukawa couplings of the quarks and leptons to $S$ and to the ordinary Standard Model Higgs field $H$, after integrating out all the fields whose mass is of order the flavor scale $M_{F}$, is assumed to be

$$
\mathcal{L}^{Y u k a w a}=-\hat{\lambda}_{i j}^{u} \epsilon^{a b} \bar{Q}_{L i}^{a} H^{\dagger b} u_{R j}-\hat{\lambda}_{i j}^{d} \bar{Q}_{L i}^{a} H^{a} d_{R j}-\hat{\lambda}_{i j}^{l} \bar{L}_{L i}^{a} H^{a} l_{R j}+\text { h.c. },
$$

where $i, j=1,2,3$ are family indices, and $a, b=1,2$ are $S U(2)_{L}$ indices. The $\hat{\lambda}$ 's are given by the following expressions.

$$
\hat{\lambda}^{u}=\left(\begin{array}{ccc}
h_{11}^{u} \hat{\epsilon}^{4} & h_{12}^{u} \hat{\epsilon}^{4} & h_{13}^{u} \hat{\epsilon}^{4} \\
h_{21}^{u} \hat{\epsilon}^{4} & h_{22}^{u} \hat{\epsilon}^{2} & h_{23}^{u} \hat{\epsilon}^{2} \\
h_{31}^{u} \hat{\epsilon}^{4} & h_{32}^{u} \hat{\epsilon}^{2} & h_{33}^{u}
\end{array}\right), \quad \hat{\lambda}^{d}=\left(\begin{array}{ccc}
h_{11}^{d} \hat{\epsilon}^{6} & h_{12}^{d} \hat{\epsilon}^{6} & h_{13}^{d} \hat{\epsilon}^{6} \\
h_{21}^{d} \hat{\epsilon}^{6} & h_{22}^{d} \hat{\epsilon}^{4} & h_{23}^{d} \hat{\epsilon}^{4} \\
h_{31}^{d} \hat{\epsilon}^{6} & h_{32}^{d} \hat{\epsilon}^{4} & h_{33}^{d} \hat{\epsilon}^{2}
\end{array}\right)
$$

The corresponding matrix for the charged leptons, $\hat{\lambda}^{l}$ is assumed to have the same form as $\hat{\lambda}^{d}$ with $h_{i j}^{d} \rightarrow h_{i j}^{l}$. In these expressions the $h_{i j}$ are all assumed to be of order unity, and the hierarchy among various masses and mixing angles therefore comes from the powers of $\hat{\epsilon}^{2}$, which is defined to be

$$
\hat{\epsilon}^{2} \equiv S / M_{F}
$$

The particular structure given in Eq. (2) is inspired by a model of Babu and Nandi [7], which has the same powers of $\hat{\epsilon}$, but where $\hat{\epsilon}=\left(H^{\dagger} H\right) / M_{F}^{2}$ rather than $S / M_{F}$ as here. Their model is not a typical flavon model, therefore. However, the pattern of powers of $\epsilon$ is quite typical of many abelian flavon models, and gives, as Babu and Nandi show (see below), an excellent fit to quark and lepton masses and CKM angles. If we call the vacuum expectation value of the flavon field $\langle S\rangle \equiv u$, then the small parameter that characterizes flavor changing is $\epsilon^{2} \equiv u / M_{F}$.

The Higgs potential is assumed to have the form 


$$
\begin{aligned}
V(H, S) & =\lambda\left(H^{\dagger} H\right)^{2}-\mu^{2}\left(H^{\dagger} H\right)+\lambda_{S}\left(S^{*} S\right)^{2}-\mu_{S}^{2}\left(S^{*} S\right) \\
& +\lambda^{\prime}\left(H^{\dagger} H S^{*} S\right)-\frac{1}{2}\left(\delta m^{2} S^{2}+\text { h.c. }\right) .
\end{aligned}
$$

The last term has been put in to give a soft breaking of the global $U(1)_{F}$ under which $S \rightarrow \mathrm{e}^{\mathrm{i} \theta} S$, and thus to give mass to the pseudoscalar part of $S$. (This global $U(1)$ may ultimately come from a local flavor symmetry that is broken at a higher scale.) The parameter $\delta m^{2}$ is the only one in the Higgs potential that can have a phase. However, one can absorb this by a phase rotation of $S$. Having done so, the VEV of $S$ is a real quantity. Minimizing this potential gives

$$
\begin{aligned}
& S=u+\frac{1}{\sqrt{2}} s_{1}+\frac{\mathrm{i}}{\sqrt{2}} s_{2}, \\
& H=\left(\begin{array}{c}
0 \\
v+\frac{1}{\sqrt{2}} h
\end{array}\right),
\end{aligned}
$$

where

$$
\begin{aligned}
& v^{2}=\left[2 \lambda_{S} \mu^{2}-\lambda^{\prime}\left(\mu_{S}^{2}+\delta m^{2}\right)\right] /\left(4 \lambda \lambda_{S}-\lambda^{\prime 2}\right), \\
& u^{2}=\left[2 \lambda\left(\mu_{S}^{2}+\delta m^{2}\right)-\lambda^{\prime} \mu^{2}\right] /\left(4 \lambda \lambda_{S}-\lambda^{\prime 2}\right),
\end{aligned}
$$

with $v \simeq 174 \mathrm{GeV}$, and $\left\langle s_{1}\right\rangle=\left\langle s_{2}\right\rangle=\langle h\rangle=0$. From Eqs. (3) and (5), we can write

$$
\hat{\epsilon}^{2}=\epsilon^{2}\left[1+\left(s_{1}+\mathrm{i} s_{2}\right) /(\sqrt{2} u)\right] .
$$

Consequently, the couplings of $s_{1}$ and $s_{2}$ to the quarks and leptons are obtained by taking in Eq. (1)

$$
\hat{\lambda}_{i j}^{f} H=\lambda_{i j}^{f} \hat{\epsilon}^{2 n_{i j}^{f}}\left(v+\frac{h}{\sqrt{2}}\right) \cong m_{i j}^{f}\left[1+\frac{n_{i j}^{f}\left(s_{1}+\mathrm{i} s_{2}\right)}{\sqrt{2} u}+\frac{h}{\sqrt{2} v}\right],
$$

where $f=u, d, l$, and where $n_{i j}^{f}$ is the power of $\hat{\epsilon}^{2}$ that appears in $\hat{\lambda}_{i j}^{f}$. It turns out that for the interesting phenomenology one can ignore the terms higher than linear in the fields $s_{1}$ and $s_{2}$ in Eq. (8). Note that the coupling 
of $h$ to the quarks and leptons will be made real and diagonal when the mass matrices $m_{i j}^{f}$ are, but that the coupling of the flavon fields $s_{1}$ and $s_{2}$ will not be made real and diagonal because of the extra factor of $n_{i j}^{f}$. This is what will give the flavor-changing and CP-violating effects that we shall be interested in. We see also that $s_{2}$ couples in the same way to quarks and leptons as $s_{1}$ does so but with a relative phase of i. This factor of $\mathrm{i}$ comes in squared in $s_{2}$ exchange and so does not lead to CP-violating effects to the order we are interested in.

Let us now look at how many parameters the model has. First, there are the large number of parameters that we have called $h_{i j}^{f}$. Because there are so many, there is no hope of making any sharp predictions. However, if we confine our ambition to making order of magnitude estimates of effects, then we can (for the most part) ignore the $h_{i j}^{f}$, since they are assumed all to be of order unity. This leaves the six parameters in the Higgs potential $\left(\lambda, \mu^{2}, \lambda_{S}, \mu_{S}^{2}, \lambda^{\prime}, \delta m^{2}\right)$, and the flavor scale $M_{F}$. These parameters can be traded for $v, m_{h}, u, m_{s_{1}}, \sin \phi, m_{s_{2}}$, and $M_{F}$. The VEV $v$ is known precisely; the mass of the ordinary higgs $m_{h}$ is known approximately; and the parameter $M_{F}$ is determined by the relation $\epsilon^{2} \equiv u / M_{F}$. (The value of $\epsilon^{2}$ is known approximately from the values of the quark and lepton mass ratios and the CKM angles.) Consequently, one is left with four free parameters: the masses of the scalar flavon $m_{s_{1}}$ and the pseudoscalar flavon $m_{s_{2}}$, the VEV $u$ of the flavon (which, as we have seen, controls the strength of the flavon couplings to matter), and the parameter $\sin \phi$ that describes the mixing between the ordinary Higgs and the scalar flavon. This mixing is described by the mass matrix

$$
\frac{1}{2}\left(\begin{array}{lll}
h & s_{1} & s_{2}
\end{array}\right)\left(\begin{array}{ccc}
4 \lambda v^{2} & 2 \lambda^{\prime} v u & 0 \\
2 \lambda^{\prime} v u & 4 \lambda_{S} u^{2} & 0 \\
0 & 0 & 2 \delta m^{2}
\end{array}\right)\left(\begin{array}{l}
h \\
s_{1} \\
s_{2}
\end{array}\right) .
$$

so that $\tan 2 \phi=\left(\lambda^{\prime} v u\right) /\left(\lambda_{s} u^{2}-\lambda v^{2}\right)$. We will call the mass eigenstates $h^{\prime}=\cos \phi h-\sin \phi s_{1}$, and $s^{\prime}=\sin \phi h+\cos \phi s_{1}$, and their masses $m_{h^{\prime}}$, and $m_{s^{\prime}}$, respectively.

Turning to the diagonalization of the quark and lepton mass matrices, one finds that

$$
\left(m_{u}, m_{c}, m_{t}\right) \cong\left(\left|h_{11}^{u}-h_{12}^{u} h_{21}^{u} / h_{22}^{u}\right| \epsilon^{6},\left|h_{22}^{u}\right| \epsilon^{2},\left|h_{33}^{u}\right|\right) v
$$




$$
\begin{aligned}
&\left(m_{d}, m_{s}, m_{b}\right) \cong\left(\left|h_{11}^{d}\right| \epsilon^{6},\left|h_{22}^{d}\right| \epsilon^{4},\left|h_{33}^{d}\right| \epsilon^{2}\right) v \\
&\left(m_{e}, m_{\mu}, m_{\tau}\right) \cong\left(\left|h_{11}^{l}\right| \epsilon^{6},\left|h_{22}^{l}\right| \epsilon^{4},\left|h_{33}^{l}\right| \epsilon^{2}\right) v
\end{aligned}
$$

and

$$
\begin{aligned}
&\left|V_{u s}\right| \cong\left|\frac{h_{12}^{d}}{h_{22}^{d}}-\frac{h_{12}^{u}}{h_{22}^{u}}\right| \epsilon^{2}, \\
&\left|V_{c b}\right| \cong\left|\frac{h_{23}^{d}}{h_{33}^{d}}-\frac{h_{23}^{u}}{h_{33}^{u}}\right| \epsilon^{2}, \\
&\left|V_{u b}\right| \cong\left|\frac{h_{13}^{d}}{h_{33}^{d}}-\frac{h_{13}^{u}}{h_{33}^{u}}-\frac{h_{12}^{u} h_{23}^{d}}{h_{22}^{u} h_{33}^{d}}+\frac{h_{12}^{u} h_{23}^{d}}{h_{22}^{u} h_{33}^{d}}\right| \epsilon^{4} .
\end{aligned}
$$

Babu and Nandi [7] showed that this gives a reasonable fit to the data. They took the data to be $m_{u}(1 \mathrm{GeV})=5.1 \mathrm{MeV}, m_{d}(1 \mathrm{GeV})=8.9 \mathrm{MeV}$, $m_{s}(1 \mathrm{GeV})=175 \mathrm{MeV}, m_{c}\left(m_{c}\right)=1.27 \mathrm{GeV}, m_{b}\left(m_{b}\right)=4.25 \mathrm{GeV}, m_{t}^{\text {phys }}=$ $175 \mathrm{GeV}, m_{\tau}=1.78 \mathrm{GeV}, m_{\mu}=105.6 \mathrm{MeV}$, and $m_{e}=511 \mathrm{keV}$. Extrapolating, using the 3-loop QCD and one-loop QED beta functions, with $\alpha_{s}\left(M_{Z}\right)=$ 0.118 , they obtained the running masses in GeV evaluated at $m_{t}: m_{t} \simeq 166$, $m_{c} \simeq 0.6, m_{u} \simeq 0.0022, m_{b} \simeq 2.78, m_{s} \simeq 0.075, m_{d} \simeq 0.0038, m_{\tau} \simeq 1.75$, $m_{\mu} \simeq 0.104$, and $m_{e} \simeq 0.0005$. These are well fit by $\epsilon^{2} \simeq(1 / 6.5)^{2} \cong 0.024$, if one takes $\left|h_{11}^{u}-h_{12}^{u} h_{21}^{u} / h_{22}^{u}\right| \simeq 0.95,\left|h_{22}^{u}\right| \simeq 0.14,\left|h_{33}^{u}\right| \simeq 0.96,\left|h_{11}^{d}\right| \simeq 1.65$, $\left|h_{22}^{d}\right| \simeq 0.77,\left|h_{33}^{d}\right| \simeq 0.68,\left|h_{11}^{l}\right| \simeq 0.21,\left|h_{22}^{l}\right| \simeq 1.06$, and $\left|h_{33}^{l}\right| \simeq 0.42$.

Note that with the exception of $h_{22}^{u}$ and $h_{11}^{l}$ all these are of order unity. And as emphasized in [7] the smallness of $h_{22}^{u}$ actually helps account for the values of $V_{u s}$ and $V_{u b}$. From Eq. (11) one sees that with $h_{22}^{u} \simeq 1 / 7$, these mixings come out to be $V_{u s} \sim 7 \epsilon^{2} \sim 0.2$, and $V_{u b} \sim 7 \epsilon^{4} \sim 3 \times 10^{-3}$.

As mentioned, in the basis where the mass matrices of the quarks and leptons are diagonal and real, the couplings of $s_{1}$ and $s_{2}$ remain with offdiagonal and complex elements, due to the extra factors of $n_{i j}^{f}$ in Eq. (8). However, it is interesting that the flavor-diagonal couplings of $s_{1}$ are, in fact, real to leading order in the small parameter $\epsilon^{2}$. That is, the imaginary part of these diagonal couplings is of order $\epsilon^{2} \simeq 0.02$ times the real part. This is significant for the lepton and quark electric dipole moments, as we shall see. The reason that the diagonal couplings of $s_{1}$ are real to leading order can be seen by looking at a simple two-by-two example: 


$$
Y_{h}=\left(\begin{array}{ll}
h_{11} \epsilon^{2 n_{11}} & h_{12} \epsilon^{2 n_{12}} \\
h_{21} \epsilon^{2 n_{21}} & h_{22} \epsilon^{2 n_{22}}
\end{array}\right), \quad Y_{s_{1}}=\left(\begin{array}{ll}
h_{11} n_{11} \epsilon^{2 n_{11}} & h_{12} n_{12} \epsilon^{2 n_{12}} \\
h_{21} n_{21} \epsilon^{2 n_{21}} & h_{22} n_{22} \epsilon^{2 n_{22}}
\end{array}\right) \frac{v}{u}
$$

In the basis where $Y_{h}$ is diagonal and real, which we shall denote by primes,

$$
\begin{aligned}
\left(Y_{h}^{\prime}\right)_{11} \cong\left[h_{11} \epsilon^{2 n_{11}}-\frac{h_{12} h_{21}}{h_{22}} \epsilon^{2\left(n_{12}+n_{21}-n_{22}\right)}\right] \mathrm{e}^{\mathrm{i} \alpha}, \\
\left(Y_{s_{1}}^{\prime}\right)_{11} \cong\left[h_{11} n_{11} \epsilon^{2 n_{11}}-\frac{h_{12} h_{21}}{h_{22}}\left(n_{12}+n_{21}-n_{22}\right) \epsilon^{2\left(n_{12}+n_{21}-n_{22}\right)}\right] \mathrm{e}^{\mathrm{i} \alpha} \frac{v}{u}
\end{aligned}
$$

The factor of $\mathrm{e}^{\mathrm{i} \alpha}$ is the phase rotation required to make $\left(Y_{h}^{\prime}\right)_{11}$ real. (In the same basis, the matrix $Y_{s_{1}}^{\prime}$ is easily seen to be non-diagonal: $\left|\left(Y_{s_{1}}^{\prime}\right)_{12}\right| \cong$ $\left|h_{12}\left(n_{12}-n_{22}\right) \epsilon^{2 n_{12}}\right|(v / u)$, and $\left|\left(Y_{s_{1}}^{\prime}\right)_{21}\right| \cong\left|h_{21}\left(n_{21}-n_{22}\right) \epsilon^{2 n_{21}}\right|(v / u)$.) In Eq. (13) one sees two terms in the expression for $\left(Y_{h}^{\prime}\right)_{11}$. There are two cases to consider: either these two terms are of the same order in $\epsilon^{2}$, or one is higher order in $\epsilon^{2}$ than the other. If they are the same order, then $n_{12}+n_{21}-n_{22}=$ $n_{11}$, which means that $\left(Y_{s_{1}}^{\prime}\right)_{11}=n_{11}\left(Y_{h}^{\prime}\right)_{11}(v / u)$, a real quantity, to leading order in $\epsilon^{2}$. If, on the other hand, one term in $\left(Y_{h}^{\prime}\right)_{11}$ is of lower order in $\epsilon^{2}$ than the other and dominates, then the corresponding term dominates in $\left(Y_{s_{1}}^{\prime}\right)_{11}$. Consequently, to leading order in $\epsilon^{2}$, one has again that $\left(Y_{s_{1}}^{\prime}\right)_{11}$ is just an integer times $\left(Y_{h}^{\prime}\right)_{11}(v / u)$ and therefore real.

This conclusion generalizes to more complicated situations. It is true for $N$-by- $N$ matrices. It is also true if there are several abelian flavon fields giving several $\epsilon$ parameters, as long as contributions to diagonal Yukawa couplings that are of different orders in the small parameters are not accidentally numerically comparable.

\section{Flavor-changing and CP-violating processes}

We are now ready to discuss various flavor-changing and CP-violating processes. The ones that shall be of chief interest are $\Delta m_{K}^{2}$ and $\epsilon_{K}$ in the neutral Kaon system, the electric dipole moment of the electron $d_{e}$, the decay $\mu \rightarrow e+\gamma$, and $\mu-e$ conversion on nuclei $\mu+N \rightarrow e+N$. It is straightforward 
to calculate the contributions to these effects coming from flavon exchange in our toy model.

The relevant couplings for flavor-changing and CP-violating processes, in the physical basis of fermions and bosons, can be parametrized as

$$
\mathcal{L}=-\frac{\sqrt{m_{i} m_{j}}}{v} \bar{f}_{i}\left(\Delta_{i j}^{a L} P_{L}+\Delta_{i j}^{a R} P_{R}\right) f_{j} H_{a}+g m_{W} \cos \varphi_{a} W^{+} W^{-} H_{a}+\cdots
$$

where $a=h^{\prime}, s^{\prime}, P_{L, R}=\left(1 \mp \gamma_{5}\right) / 2$, and where indices $i$ and $j$ run over all quarks and charged leptons. We observe that due to the scalar nature of $h^{\prime}$ and $s^{\prime}$, to the leading order in $\epsilon^{2}, \Delta_{i i}^{a}{ }^{L} \equiv \Delta_{i i}^{a} R *=\Delta_{i i}^{a} R \equiv \Delta_{i i}^{a}$ is real for all i's. (See the discussion after Eq. (13).) Acting on Yukawa coupling matrices with a set of bi-unitary transformations that brings fermion mass matrices into diagonal form, and simultaneously diagonalizing the Higgs sector one finds that

$$
\begin{aligned}
& \Delta_{e e}^{h^{\prime} L}=4 \chi^{2} \epsilon^{2} \frac{h_{12}^{l} h_{21}^{l}}{\sqrt{2}} \sin \phi \frac{v}{u}, \quad \Delta_{e e}^{h^{\prime} R}=4 \chi^{2} \epsilon^{2} \frac{h_{12}^{l *} h_{21}^{l *}}{\sqrt{2}} \sin \phi \frac{v}{u} \\
& \Delta_{e \mu}^{h^{\prime} L}=-\chi \epsilon \frac{h_{12}^{l}}{\sqrt{2}} \sin \phi \frac{v}{u}, \quad \Delta_{e \mu}^{h^{\prime} R}=-\chi \epsilon \frac{h_{21}^{l *}}{\sqrt{2}} \sin \phi \frac{v}{u},
\end{aligned}
$$

and

$$
\cos \varphi_{h^{\prime}}=\cos \phi, \quad \cos \varphi_{s^{\prime}}=\sin \phi,
$$

where we have omitted a term in $\Delta_{e e}$ which is real and leading order in $\epsilon^{2}$, and introduced $\chi=\left(\left|h_{11}^{l}\right|\left|h_{22}^{l}\right|\right)^{-1 / 2}$. The coefficients $\Delta_{i j}^{s^{\prime} L, R}$ are obtained from $\Delta_{i j}^{h^{\prime} L, R}$ by making the transformation $\cos \phi \rightarrow \sin \phi$, and $\sin \phi \rightarrow-\cos \phi$.

The electric dipole moment of the electron $\left(d_{e}\right)$ comes from the familiar type of two-loop graph [8] shown in Fig. 11. In terms of the original fields $s_{1}$, $s_{2}$ and $h$ coming from $S$ and $H$, rather than the mass eigenstates, one sees that the field that couples to the $W$ or $t$ loop must be $h$. This can be seen as follows. The $s_{i}$ have no coupling to $t$ at the leading order in $\epsilon^{2}$, since the $t$ mass comes from order $\left(S / M_{F}\right)^{0}$. i.e. $n_{33}^{u}=0$. (See Eqs. (2) and (8).) The $s_{i}$ also have no coupling to the $W^{ \pm}$since $S$ does not participate in breaking $S U(2)_{L} \times U(1)_{Y}$. (If there were two Higgs doublets in the model, then the heavy loop could be a charged Higgs, in which case the field coupling to it in Fig. 1 could be an $s_{1}$.) However, the field coupling to the electron line 
must be either $s_{1}$ or $s_{2}$ in order to obtain a CP-violating phase, since the couplings of $h$ are real and flavor diagonal in the physical basis of the leptons. However, the $s_{2}$, while it can give a CP-violating phase, does not mix with the $h$ and therefore would not be able to attach to the $W$ or $t$ loop. The scalar line in the two-loop graph for $d_{e}$ is thus $s_{1}$ where it attaches to the electron, and $h$ where it attaches to the $W$ or $t$ loop. Consequently, the electron edm is proportional to the mixing $\sin \phi \cos \phi$. A significant point about the $d_{e}$ diagram, which has already been alluded to, is that while the $s_{1}$ coupling to the electron has a CP-violating phase, that phase brings in an extra suppression of order $\epsilon^{2}$ (see Eq. (15)). The electric dipole moment of a charged lepton is given by

$$
d_{i}=\frac{e G_{F} \alpha}{8 \sqrt{2} \pi^{3}} m_{i} \operatorname{Im}\left[A_{i i}^{L}-A_{i i}^{R}\right]
$$

where the dominant, reduced amplitude [9], comes from $W$ loop and reads

$$
A_{i j}^{L, R}=-\sum_{a} \cos \varphi_{a} \Delta_{i j}^{a L, R}\left[3 f\left(\frac{m_{W}^{2}}{m_{a}^{2}}\right)+\frac{23}{4} g\left(\frac{m_{W}^{2}}{m_{a}^{2}}\right)+\frac{3}{4} h\left(\frac{m_{W}^{2}}{m_{a}^{2}}\right)\right] .
$$

Setting $h_{12}^{l}=h_{21}^{l}=\mathrm{e}^{-\mathrm{i} \pi / 4}$ in Eq. (15), the electron edm comes out to be

$$
d_{e}=\left(1.5 \times 10^{-27} e \mathrm{~cm}\right) \sin \phi \cos \phi\left(\frac{v}{u}\right)\left[F\left(\frac{m_{W}^{2}}{m_{h^{\prime}}^{2}}\right)-F\left(\frac{m_{W}^{2}}{m_{s^{\prime}}^{2}}\right)\right]
$$

where $F(z) \equiv 3 f(z)+\frac{23}{4} g(z)+\frac{3}{4} h(z)$, and the functions $f, g$, and $h$ are as defined in [9] in Eqs. (10), (11), and (15), respectively. The function $F(z)$ is plotted in Fig. 2. Using the experimental value $d_{e}=0.18 \times 10^{-26} \mathrm{ecm}$ [10 gives the following limit

$$
\sin \phi \cos \phi\left(\frac{v}{u}\right)\left[F\left(\frac{m_{W}^{2}}{m_{h^{\prime}}^{2}}\right)-F\left(\frac{m_{W}^{2}}{m_{s^{\prime}}^{2}}\right)\right] \leq 1.2 .
$$

The diagram for $\mu \rightarrow e+\gamma$ is of the same two-loop type as the electron edm diagram, except that one of the external leptons is a $\mu$ rather than an $e$. As in the $d_{e}$ case, the scalar which couples to the lepton line must be $s_{1}$ (here because it involves flavor-changing), while the scalar that couples to the $W^{ \pm}$or $t$ loop must be $h$. Thus the amplitude here is also proportional to $\sin \phi \cos \phi$. The branching ratio for the process $l_{j} \rightarrow l_{i}+\gamma$ is given by 


$$
B\left(l_{j} \rightarrow l_{i}+\gamma\right)=\frac{3}{4}\left(\frac{\alpha}{\pi}\right)^{3} \frac{m_{i}}{m_{j}}\left(\frac{1}{2}\left|A_{i j}^{L}\right|^{2}+\frac{1}{2}\left|A_{i j}^{R}\right|^{2}\right) .
$$

Again setting $h_{12}^{l}=h_{21}^{l}=\mathrm{e}^{-\mathrm{i} \pi / 4}$, and imposing the experimental limit $B(\mu \rightarrow$ $e+\gamma) \leq 1.2 \times 10^{-11}$ [11], one obtains

$$
\sin \phi \cos \phi\left(\frac{v}{u}\right)\left[F\left(\frac{m_{W}^{2}}{m_{h^{\prime}}^{2}}\right)-F\left(\frac{m_{W}^{2}}{m_{s^{\prime}}^{2}}\right)\right] \leq 2.2 .
$$

The diagram relevant for $\mu$-e conversion on nuclei is Fig. 3. The field that couples to the lepton line must be $s_{1}$ or $s_{2}$, but the field that couples at the quark line may be $h, s_{1}$, or $s_{2}$. It is well known that the contributions of the pseudoscalar exchange to the coherent $\mu-e$ conversion on nuclei can be neglected [12] and will be ignored in our calculations. The contributions to the amplitude from diagrams where the scalar couples to the lepton as $s_{1}$ but to the quark as $h$ go as $\sin \phi \cos \phi\left(1 / m_{h^{\prime}}^{2}-1 / m_{s^{\prime}}^{2}\right)$. Those in which the scalar couples to both the lepton line and the quark line as $s_{1}$ go as $\cos ^{2} \phi\left(1 / m_{s^{\prime}}^{2}\right)+\sin ^{2} \phi\left(1 / m_{h^{\prime}}^{2}\right)$. We shall see these expressions emerge in the formulas that appear below.

The branching ratio of $\mu$-e conversion $B\left(\mu^{-}+(A, Z) \rightarrow e^{-}+(A, Z)\right)$, defined to be the ratio of decay widths $\Gamma\left(\mu^{-}+(A, Z) \rightarrow e^{-}+(A, Z)\right) / \Gamma\left(\mu^{-}+\right.$ $(A, Z) \rightarrow$ capture $)$, can be found using the procedure outlined in [13, 14. We obtain

$$
B=2 G_{F}^{2} m_{e} m_{\mu} \frac{\alpha^{3} m_{\mu}^{5} Z_{e f f}^{4}}{\pi^{2} Z \Gamma_{c a p t}} A^{2} F\left(q^{2}\right)^{2}\left[\left|\sum_{a} \frac{\tilde{m}_{N}^{a}}{m_{a}^{2}} \Delta_{e \mu}^{a L}\right|^{2}+\left|\sum_{a} \frac{\tilde{m}_{N}^{a}}{m_{a}^{2}} \Delta_{e \mu}^{a R}\right|^{2}\right],
$$

where $F\left(q^{2}\right)$ is the nucleon form factor, $Z_{\text {eff }}$ is the effective atomic number, and where $\tilde{m}_{N}^{a}$ contains the heavy quark effects in effective scalar-nucleonnucleon coupling [15] and is given by

$$
\tilde{m}_{N}^{a}=\left\langle N\left|\sum_{l=u, d, s} m_{l} \Delta_{l l}^{a} \bar{f}_{l} f_{l}+\sum_{h=t, b, c} m_{h} \Delta_{h h}^{a} \bar{f}_{h} f_{h}\right| N\right\rangle .
$$

We derive the most general, model independent, expression for $\tilde{m}_{N}^{a}$ using the approach of Shifman et. al. [16], and subsequent improvements of inclusion of strange and heavy quark contributions discussed in [17, 18] as followst]

\footnotetext{
${ }^{1}$ Our general expression for $\tilde{m}_{N}^{a}$ reproduces Eq. (3) of Ref. [15] but yields an additional term in Eq. (20) where authors analyze MSSM model. The additional piece is $\sigma_{\pi N}(\cot \beta+$ $\tan \beta) / 2$.
} 


$$
\tilde{m}_{N}^{a}=\left(\sum_{h} \Delta_{h h}^{a}\right) \frac{2}{27}\left[m_{N}-\sigma_{\pi N}\left(1+\frac{y}{2} \frac{m_{s}}{\bar{m}}\right)\right]+\sigma_{\pi N}\left[\frac{\Delta_{u u}^{a}+\Delta_{d d}^{a}}{2}+\Delta_{s s}^{a} \frac{y}{2} \frac{m_{s}}{\bar{m}}\right]
$$

where $h$ runs over heavy quarks $(t, b, c), y=2\langle N|\bar{s} s| N\rangle /\langle N|\bar{u} u+\bar{d} d| N\rangle$ is the strange content in the nucleon, $\sigma_{\pi N}$ is the pion-nucleon sigma term, $m_{N}$ is the nucleon mass, and $\bar{m}=\left(m_{u}+m_{d}\right) / 2$. In our model, the diagonalization procedure in quark sector, to the leading order in $\epsilon^{2}$, leads to

$$
\Delta_{i i}^{h^{\prime}}=\left[\cos \phi-\kappa_{i} \frac{v}{u} \sin \phi\right] / \sqrt{2},
$$

where $\left(\kappa_{u}, \kappa_{c}, \kappa_{t}, \kappa_{d}, \kappa_{s}, \kappa_{b}\right)=(3,1,0,3,2,1)$. Note that $\kappa_{i}$ 's are the powers of $\hat{\epsilon}^{2}$ of the appropriate diagonal elements that appear in $\hat{\lambda}_{i j}^{f}$ of Eq. (2).

For $\mu$-e conversion on ${ }_{22}^{48} \mathrm{Ti}$, we set $Z_{\text {eff }}=17.6, F\left(q^{2}=-m_{\mu}^{2}\right)=0.54$, $\Gamma_{\text {capt }}=2.59 \times 10^{6} \mathrm{~s}^{-1}\left[19\right.$, impose the experimental limit $B<4.3 \times 10^{-12}$ 20], take $\bar{m}=5 \mathrm{MeV}$, and use the set $\left(y, \sigma_{\pi N}\right)=(0.47,60 \mathrm{MeV})$ [17], to obtain

$$
\left(\frac{v}{u}\right)\left|\sin \phi \cos \phi\left(\frac{1}{m_{h^{\prime}}^{2}}-\frac{1}{m_{s^{\prime}}^{2}}\right) m-\left(\frac{v}{u}\right)\left(\frac{\sin ^{2} \phi}{m_{h^{\prime}}^{2}}+\frac{\cos ^{2} \phi}{m_{s^{\prime}}^{2}}\right) m^{\prime}\right| \leq \frac{9 \times 10^{-5}}{\mathrm{GeV}}
$$

where $m \simeq 350 \mathrm{MeV}$, and $m^{\prime} \simeq 500 \mathrm{MeV}$.

The diagram relevant for the $\Delta S=2$ processes is Fig. 田. Here, the field that couples at both quark lines must be $s_{1}$ or $s_{2}$. Thus there are contributions that go as $\cos ^{2} \phi\left(1 / m_{s^{\prime}}^{2}\right)+\sin ^{2} \phi\left(1 / m_{h^{\prime}}^{2}\right)$ and as $1 / m_{s_{2}}^{2}$. Noting that $\Delta_{d s}^{L, R}$ is obtained from $\Delta_{e \mu}^{L, R}\left(\right.$ Eq. (15)) by replacing $h_{i j}^{l}$ with $h_{i j}^{d}$, and using the vacuum saturation approximation for the hadronic element [21], we find a new contribution coming from the scalar exchange to be

$$
\begin{aligned}
\epsilon_{K}^{a} & \simeq \frac{C_{K}}{m_{a}^{2}}\left\{\left(\frac{1}{6} \frac{M_{K}^{2}}{\left(m_{d}+m_{s}\right)^{2}}+\frac{1}{6}\right) \operatorname{Im}\left[\left(\frac{h_{12}^{d *}+h_{21}^{d}}{\sqrt{2}}\right)^{2}\right]\right. \\
& \left.-\left(\frac{11}{6} \frac{M_{K}^{2}}{\left(m_{d}+m_{s}\right)^{2}}+\frac{1}{6}\right) \operatorname{Im}\left[\left(\frac{h_{12}^{d *}-h_{21}^{d}}{\sqrt{2}}\right)^{2}\right]\right\}\left(1-\cos ^{2} \varphi_{a}\right),
\end{aligned}
$$

while the exchange of pseudoscalar $s_{2}$, due to the extra factor of i, yields 


$$
\begin{aligned}
\epsilon_{K}^{s_{2}} & \simeq \frac{C_{K}}{m_{s_{2}}^{2}}\left\{\left(\frac{1}{6} \frac{M_{K}^{2}}{\left(m_{d}+m_{s}\right)^{2}}+\frac{1}{6}\right) \operatorname{Im}\left[\left(\frac{h_{12}^{d *}-h_{21}^{d}}{\sqrt{2}}\right)^{2}\right]\right. \\
& \left.-\left(\frac{11}{6} \frac{M_{K}^{2}}{\left(m_{d}+m_{s}\right)^{2}}+\frac{1}{6}\right) \operatorname{Im}\left[\left(\frac{h_{12}^{d *}+h_{21}^{d}}{\sqrt{2}}\right)^{2}\right]\right\},
\end{aligned}
$$

where we introduce

$$
C_{K}=\frac{f_{K}^{2} M_{K} B_{K} \epsilon^{12}}{8 \sqrt{2} \Delta M_{K}}\left(\frac{v}{u}\right)^{2} .
$$

Using $B_{K}=0.75, \Delta M_{K} \simeq 3.49 \times 10^{-12} \mathrm{MeV}, f_{K} \simeq 160 \mathrm{MeV}, M_{K} \simeq 497.67$ $\mathrm{MeV}, m_{s}=175 \mathrm{MeV}, m_{d}=8.9 \mathrm{MeV}, h_{12}^{d}=h_{21}^{d}=\mathrm{e}^{-\mathrm{i} \pi / 4}$, and requiring the terms involving $m_{h^{\prime}}, m_{s^{\prime}}$, and $m_{s_{2}}$ separately to contribute to $\epsilon_{K}$ an amount less than the experimental value of that quantity $\left(\left|\epsilon_{K}\right|=2.26 \times 10^{-3}\right.$ [10]) give the limits

$$
\left(\frac{v}{u}\right)^{2} \frac{\sin ^{2} \phi}{m_{h^{\prime}}^{2}},\left(\frac{v}{u}\right)^{2} \frac{\cos ^{2} \phi}{m_{s^{\prime}}^{2}} \leq \frac{3.9 \times 10^{-6}}{\mathrm{GeV}^{2}}, \quad\left(\frac{v}{u}\right)^{2} \frac{1}{m_{s_{2}}^{2}} \leq \frac{3.8 \times 10^{-5}}{\mathrm{GeV}^{2}}
$$

If we take $m_{h^{\prime}} \simeq 10^{2} \mathrm{GeV}$, as suggested by experiment, then Eq. (31) implies that $(v / u) \sin \phi \leq \frac{1}{5}$, which is not a very stringent bound. Substituting this into Eq. (19), one sees that the electron edm can easily be near the present published experimental limit. For instance, taking $(v / u) \sin \phi \simeq 0.1$, so that flavon exchange contributes of order $1 / 5$ of the experimental value of $\epsilon_{K}$, and taking $m_{s^{\prime}} \simeq 300 \mathrm{GeV}$, Eq. (14) gives $d_{e} \sim\left(0.6 \times 10^{-27} e \mathrm{~cm}\right) \cos \phi$.

Comparing Eqs. (20) and (22) (in which the unknown parameters, $\sin \phi$, $m_{s^{\prime}}$, and $u$, enter in exactly the same way) reveals that the present limits on the decay $\mu \rightarrow e+\gamma$ and the electron edm are about equally sensitive to flavon exchange in this model. For example, if the CP-violating phases are large and all $h_{i j}$ are close to one, as was assumed in deriving Eqs. (19) and (22), and $d_{e}$ is just below the present limit, then the rate for $\mu \rightarrow e+\gamma$ is roughly a forth of the present limit.

One sees here the importance of the fact that the diagonal Yukawa couplings of the flavon field $s_{1}$ have phases suppressed by $\epsilon^{2} \simeq 2 \times 10^{-2}$. Were it not so, then the present limit on the electron edm would imply that the rate for $\mu \rightarrow e+\gamma$ was at least four orders of magnitude below present 
limits (unless parameters were fine-tuned). $\mu+N \rightarrow e+N$ would also be suppressed.

Turning to $\mu-e$ conversion on nuclei, one sees from Eq. (27), that the present limit on this is also, for a wide range of parameters, about as sensitive to flavon-exchange as are the present limits on $d_{e}$ and $\mu \rightarrow e+\gamma$. For example, if $(v / u) \sin \phi \simeq \frac{1}{5}$, then the first term on the left-hand side of Eq. (27) (i.e. the term proportional to $\left.\sin \phi \cos \phi / m_{h_{0}}^{2}\right)$ gives a contribution to the rate for $\mu-e$ conversion that is about an order of magnitude below the present limit. However, in some regions of parameter space, $\mu+N \rightarrow e+N$ can be the most sensitive to flavon exchange. Suppose, for example, that $v / u$ is smaller, but not much smaller, than one, and that $\sin \phi \ll 1$. Then both $d_{e}$ and $\mu \rightarrow e+\gamma$ are highly suppressed, whereas $\mu+N \rightarrow e+N$ need not be because of the term that goes as $\frac{\cos ^{2} \phi}{m_{s^{\prime}}^{2}}$ on the left-hand side of Eq. (27).

We have only considered the effects arising from the effective Yukawa terms in Eq. (1). However, there is another source of flavor violation from flavon exchange that can be very important. To get the effective low energy Yukawa terms in Eq. (1), fermions having mass of order $M_{F}$ are integrated out. There are diagrams involving these heavy fermions that can be important. The most important such diagram is that shown in Fig. 5, which is a contribution to $K-\bar{K}$ mixing. The internal fermion has mass of order $M_{F}$. The external fermion is the $s_{0}$ quark, i.e. the $s$ quark in the original basis in which the Yukawa matrices of Eq. (2) are written. When one goes to the physical basis of the light quarks, $s_{0}$ will contain a small admixture of the physical $d$ quark: $s_{0}=s+O\left(\epsilon^{2}\right) d$. Consequently, there will be from Fig. 5 a $\Delta S=2$ piece that goes as $\epsilon^{4}$. The Yukawa couplings in Fig. 5 may be assumed to be of order unity. (The only reason the effective Yukawa couplings of the known light quarks are small is that they are suppressed by powers of $\epsilon^{2}$, since they arise from integrating out heavy fermions. However, in the underlying theory containing those heavy fermions there is no reason for the Yukawa couplings to be small.) The coefficient of the $\Delta S=2$ operator arising from Fig. 5 should therefore typically be of order $\left(16 \pi^{2}\right)^{-1} \epsilon^{4}\left(1 / M_{F}^{2}\right)=\left(16 \pi^{2}\right)^{-1} \epsilon^{8} u^{-2}$. Using $\epsilon^{2} \sim 2 \times 10^{-2}$ and $u \sim 300$ $\mathrm{GeV}$, one has that the coefficient of the $\Delta S=2$ term is of order $10^{-14} \mathrm{GeV}^{-2}$. With some of the phases or couplings being assumed somewhat smaller than one, the contribution from Fig. 5 can easily be within the limit set by $\epsilon_{K}$. 


\section{Conclusions}

We presented a simple flavon model that can accommodate the observed hierarchy of the charged fermion masses and mixings in terms of the powers of one small parameter. It has been shown that the flavor-diagonal couplings of the flavon field, under a general set of assumptions, are real to the leading order in that parameter. This implies that flavor changing and $\mathrm{CP}$ violating signatures, $d_{e}, \mu \rightarrow e+\gamma$, and $\mu$-e conversion on nuclei, can be equally near the present experimental limits with all other low energy constraints satisfied. For significant range of parameters $\mu-e$ conversion can be the most sensitive place to look for such signatures.

\section{References}

[1] C. D. Froggatt and H. B. Nielsen, Nucl. Phys. B 147, 277 (1979).

[2] Z. G. Berezhiani, Phys. Lett. B 129, 99 (1983); S. Dimopoulos, Phys. Lett. B 129, 417 (1983).

[3] See for instance: Z. Berezhiani and Z. Tavartkiladze, Phys. Lett. B 409, 220 (1997) hep-ph/9612232; N. Irges, S. Lavignac and P. Ramond, Phys. Rev. D 58, 035003 (1998) hep-ph/9802334; G. Altarelli and F. Feruglio, Phys. Lett. B 451, 388 (1999) hep-ph/9812475; Q. Shafi and Z. Tavartkiladze, Phys. Lett. B 459, 563 (1999) [hep-ph/9904249]; I. Gogoladze and A. Perez-Lorenzana, hep-ph/0112034.

[4] Y. Nir and N. Seiberg, Phys. Lett. B 309, 337 (1993) hhep-ph/9304307; M. Leurer, Y. Nir and N. Seiberg, Nucl. Phys. B 420, 468 (1994) hep$\mathrm{ph} / 9310320$.

[5] L. E. Ibanez and G. G. Ross, Phys. Lett. B 332, 100 (1994) hepph/9403338]; P. Binetruy and P. Ramond, Phys. Lett. B 350, 49 (1995) hep-ph/9412385]; V. Jain and R. Shrock, Phys. Lett. B 352, 83 (1995) hep-ph/9412367]; E. Dudas, S. Pokorski and C. A. Savoy, Phys. Lett. B 356, 45 (1995) hhep-ph/9504292]; Y. Nir, Phys. Lett. B 354, 107 (1995) hep-ph/9504312;

[6] L. Wolfenstein, Phys. Rev. Lett. 51, 1945 (1983). 
[7] K. S. Babu and S. Nandi, Phys. Rev. D 62, 033002 (2000) hepph/9907213.

[8] S. M. Barr and A. Zee, Phys. Rev. Lett. 65, 21 (1990) [Erratum-ibid. 65, $2920(1990)]$.

[9] D. Chang, W. S. Hou and W. Y. Keung, Phys. Rev. D 48, 217 (1993) hep-ph/9302267.

[10] D. E. Groom et al. [Particle Data Group Collaboration], Eur. Phys. J. C 15, 1 (2000).

[11] M. L. Brooks et al., MEGA Collaboration, Phys. Rev. Lett. 83, 1521 (1999) [hep-ex/9905013.

[12] For a review see: Y. Kuno and Y. Okada, Rev. Mod. Phys. 73, 151 (2001) hep-ph/9909265.

[13] G. Feinbeg and S. Weinberg, Phys. Rev. Lett. 3, 111, (1959) [Erratumibid. 3, 244 (1959)].

[14] W. J. Marciano and A. I. Sanda, Phys. Rev. Lett. 38, 1512 (1977).

[15] D. Ng and J. N. Ng, Phys. Lett. B 320, 181 (1994) hep-ph/9308352.

[16] M. A. Shifman, A. I. Vainshtein and V. I. Zakharov, Phys. Lett. B 78, 443 (1978).

[17] T. P. Cheng, Phys. Rev. D 38, 2869 (1988).

[18] J. Gasser, H. Leutwyler and M. E. Sainio, Phys. Lett. B 253, 252 (1991).

[19] T. Suzuki, D. F. Measday and J. P. Roalsvig, Phys. Rev. C 35, 2212 (1987).

[20] C. Dohmen et al. [SINDRUM II Collaboration.], Phys. Lett. B 317, 631 (1993).

[21] D. Atwood, L. Reina and A. Soni, Phys. Rev. D 55, 3156 (1997) hepph/9609279. 


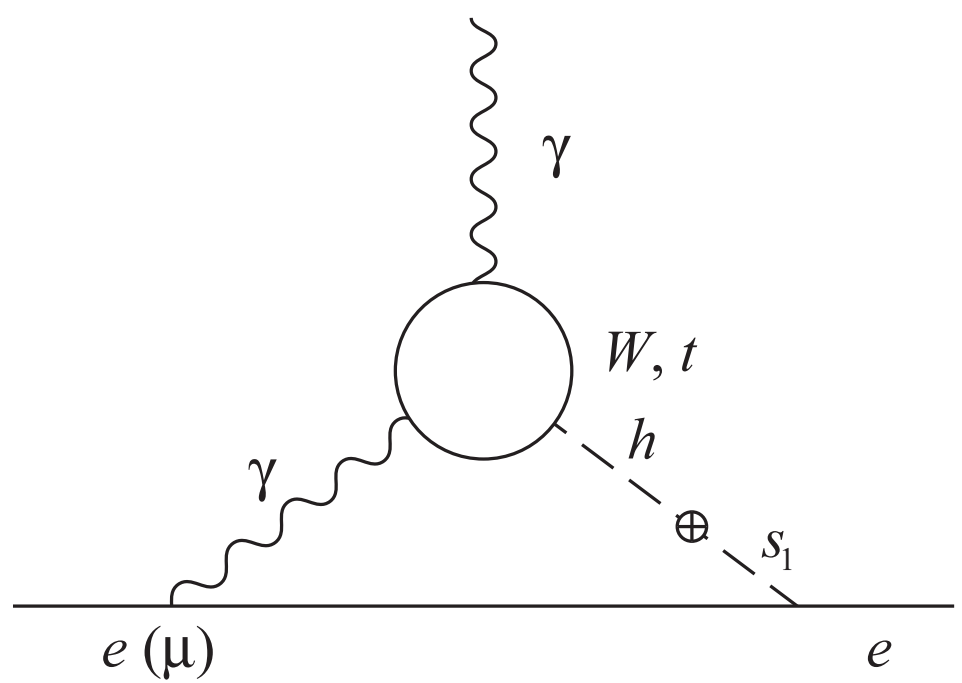

Figure 1: A two-loop Feynman diagram for electron electric dipole moment $(\mu \rightarrow e+\gamma)$.

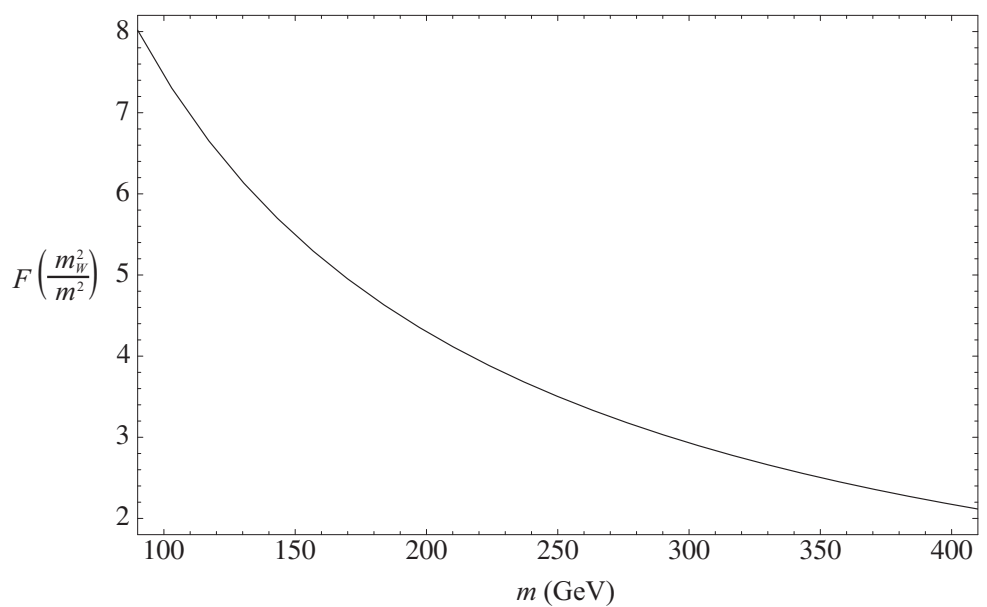

Figure 2: Plot of $F\left(m_{W}^{2} / m^{2}\right) \equiv 3 f\left(m_{W}^{2} / m^{2}\right)+\frac{23}{4} g\left(m_{W}^{2} / m^{2}\right)+\frac{3}{4} h\left(m_{W}^{2} / m^{2}\right)$ as a function of scalar mass $m$. The functions $f, g$, and $h$ are as defined in [9] in Eqs. (10), (11), and (15), respectively. 


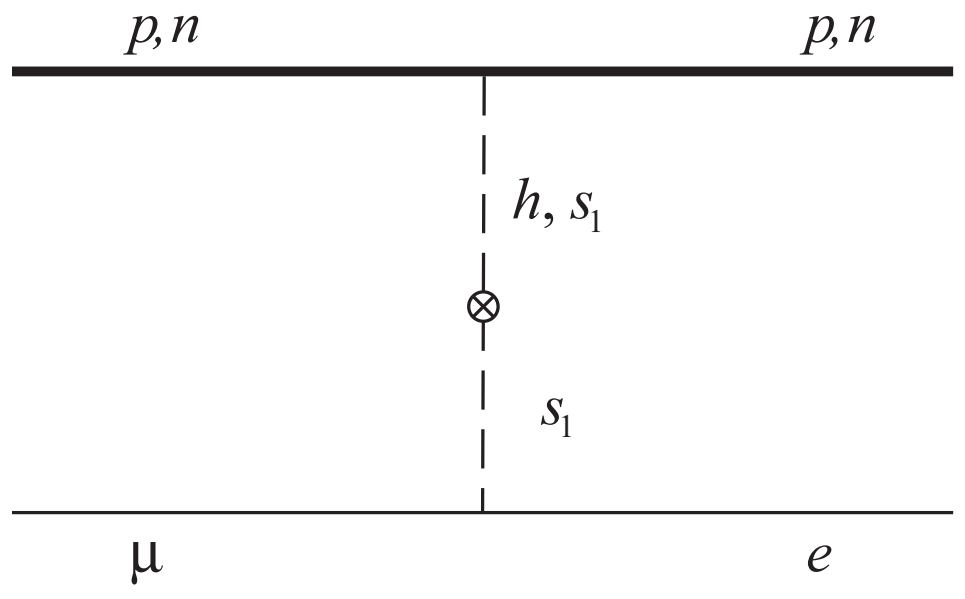

Figure 3: Tree level scalar exchange Feynman diagram for $\mu$-e conversion on nuclei.

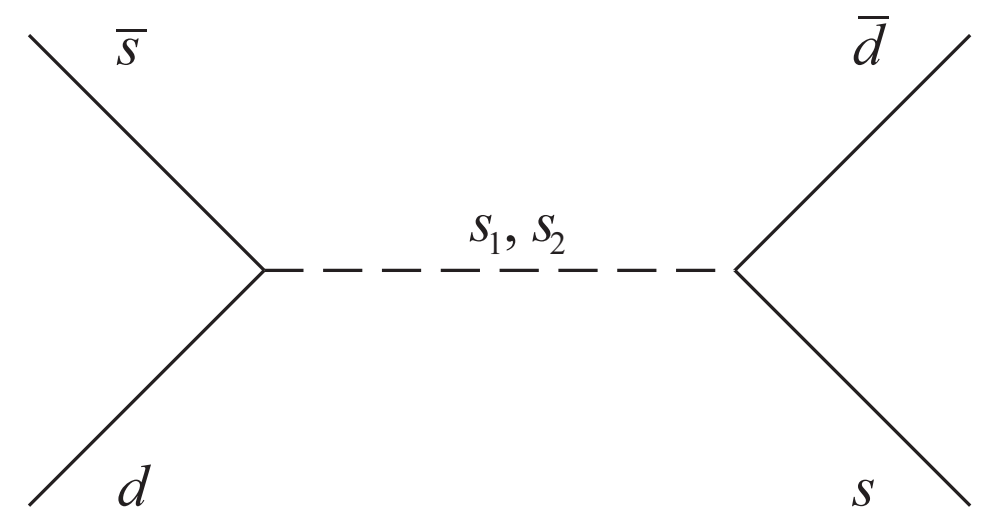

Figure 4: Tree level contribution to $K^{0}-\bar{K}^{0}$ mixing. 


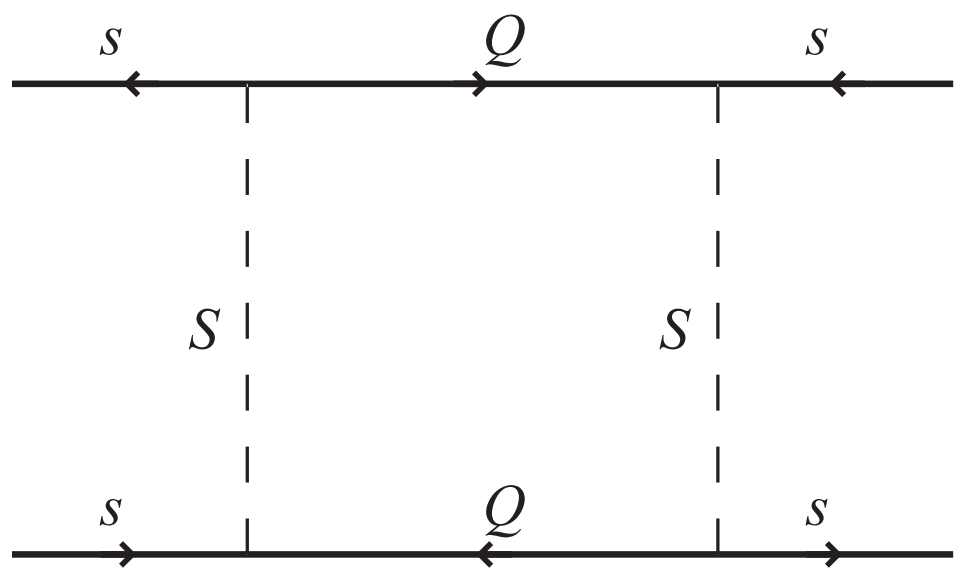

Figure 5: Box diagram contribution to $K-\bar{K}$ mixing. The internal fermion $Q$ has mass of order $M_{F}$. 